\title{
Stress found to be factor in ovarian cancer
}

Chronic psychological stress exacerbates the growth and spread of ovarian cancer in a mouse model, an effect that commonly prescribed heart medications can cancel out. If the same holds true for humans, behavioral or pharmacological interventions may someday be used to slow the progression of this often-deadly disease.

Ovarian cancer is the eighth most common type of cancer among American women. Its vague and nonspecific symptoms make it notoriously tricky to diagnose; indeed, in more than half of cases diagnosis is delayed until the disease has already spread beyond the ovary, making it difficult, if not impossible, to treat. A better understanding of the mechanisms underlying disease progression would go a long way to solving this problem.

Several years ago, Anil K. Sood of the University of Texas M.D. Anderson Cancer Center (Houston, TX) and Susan Lutgendorf of the University of Iowa (Iowa City, IA) found that ovarian cancer patients who reported higher distress levels had elevated circulating levels of vascular endothelial growth factor (VEGF) compared to women under less stress.
This signaling protein is involved in the formation of new blood vessels and plays an important role in the angiogenesis necessary for tumor growth and spread. To determine if stress levels were somehow affecting cancer progression, Sood's team turned to a mouse model for answers.

In a new study published in the August issue of Nature Medicine, Sood and his fellow researchers first sought to mimic chronic high stress conditions by immobilizing mice in a small enclosure for 2 or 6 hours daily. This caused significant increases in levels of the 'fight-or-flight' hormones norepinephrine and corticosterone. The researchers then inoculated chronically 'stressed' and 'unstressed' nude mice with human ovarian cancer cells. Three weeks later, not only did stressed mice show 2-3 fold elevations in VEGF, but they had more than twice the number of tumor nodules than controls. In addition, in more than half of the stressed mice the cancer had spread to the liver or spleen, while it remained confined to the peritoneal cavity in all of the unstressed animals.

After determining that 17 of the 19 ovarian cancer cell lines tested expressed stress

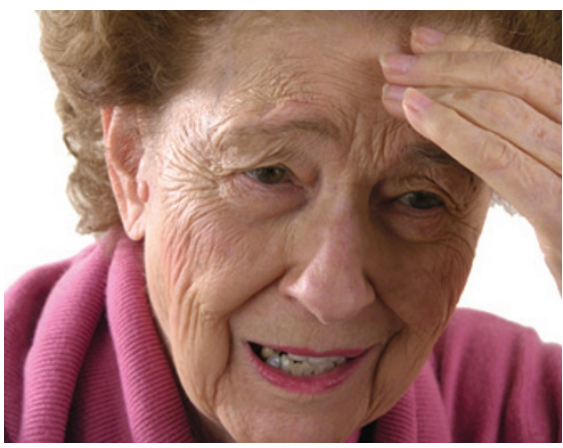

hormone receptors (which, when activated tell the cell to produce VEGF), Sood's team examined the consequences of blocking these $\beta$-adrenergic receptors. In mice treated with the ' $\beta$-blocker' propranolol, the drug seemed to nullify the effects of the stress on cancer progression.

Building on these promising results, Sood tells Lab Animal that his team now "intends to investigate other biological pathways in the context of cancer that could be affected by chronic stress" and to look at "how frequently these receptors are expressed by human ovarian cancers."

\section{Tanja Schub}

\section{IMMUNE RECEPTOR MAY BE KEY TO HAY FEVER TREATMENT}

A recent study of immune system receptors in mice may lead to the development of new clinical treatments for some immune disorders, including hay fever, asthma, and eczema.

To protect the host, the immune system must recognize and react to invading pathogens. Sometimes, however, the immune system overreacts to nonthreatening environmental substances or to host tissues, causing allergies and autoimmune disorders, respectively. Many of these 'hypersensitivity reactions' are mediated by type 2 T-helper $\left(T_{h} 2\right)$ cells; instances of $T_{h}$ 2-induced hypersensitivity may be alleviated if a type $1 \mathrm{~T}$-helper $\left(T_{h} 1\right)$ cell response is induced instead.

In research published in the August issue of Nature Immunology, a team of researchers led by Shizuo Akira at Osaka University in Japan may have found a way to 'turn on' the $T_{h} 1$ response in lieu of the $T_{h} 2$. They made this discovery during their investigation of the in vivo function of the toll-like receptor 5 (TLR5). TLRs are proteins found on many immune cells that help to activate those cells in response to certain pathogens. TLR5 receptors, which are commonly found in the intestine, are activated by recognition of the protein flagellin, the main component of bacterial flagella.
Akira's team created Tlr5-knockout mice and then used microarray analysis to compare the cytokine expression profiles between the intestinal cells in the knockout and wild-type mice after exposure to flagellin. The researchers focused on a particular type of cells called CD11 $\mathrm{c}^{+}$lamina propria cells (LPCs), which are located just beneath the intestinal epithelium. They found that the wild-type murine CD11 $\mathrm{C}^{+}$LPCs (which express TRL5) produced the pro-inflammatory cytokine IL-12, which is essential for inducing a $T_{h} 1$ immune response; the knockout mice failed to produce such a response, leading the researchers to conclude that flagellin was able to induce TLR5-mediated IL-12 secretion in the CD11 $\mathrm{C}^{+}$LPCs.

According to the paper's first author, Satoshi Uematsu, these findings support the possibility that CD11 $\mathrm{C}^{+} \mathrm{LPCs}$ might serve as targets for immunostimulatory therapies. "Having shown that CD11 $c^{+}$LPCs induce inflammatory responses via TLR5," Uematsu tells Lab Animal, "we want to try and stimulate these cells with flagellin in vivo in order to induce a $\mathrm{T}_{\mathrm{h}} 1$ immune response for treatment of hay fever or cancer immune therapy." To this end, Uematsu asserts that he and his colleagues now are trying to isolate human LPCs. Owen Young 